\title{
Financiamento das universidades líderes nos rankings internacionais, um caminho para as universidades públicas brasileiras?
}

\author{
Financing of the leading universities in \\ international rankings, a path for Brazilian public universities?
}

Felipe Tumenas ${ }^{1}$

\begin{abstract}
Resumo: As universidades públicas brasileiras enfrentam desafios no seu financiamento. Cortes e contingenciamentos vêm ao mesmo tempo de uma maior cobrança por pesquisas, internacionalização e desempenho nos rankings internacionais. Propostas para a solução desse dilema se baseiam em ideias isoladas de financiamento de universidades internacionais. Este artigo levanta as principais fontes de receitas das universidades líderes nos rankings internacionais. Através da análise dos relatórios anuais do período de 2014 a 2018, é possível ver que não existe um padrão único de financiamento nessas universidades. Ademais, a fonte de receita com maior relevância dessas universidades é pesquisa e grande parte da receita de pesquisa vem de financiamento público, o que indica que qualquer solução para a questão do financiamento das universidades deve incluir uma participação ativa do estado e que a busca de possíveis caminhos de financiamento em instituições internacionais deve ser feita com extrema cautela.
\end{abstract}

Palavras-chave: Gestão universitária. Rankings universitários. Financiamento universitário. 
Abstract: Brazilian public universities face challenges in their funding. Budget cuts come together with increased demand for research, internationalization and better performance in international rankings. Proposals for solving this dilemma are based on isolated funding ideas from international universities. This article lists the main revenue sources from the leading universities in international rankings. Looking at the annual reports from 2014 to 2018, we can see that there is no single financing pattern at these universities. Moreover, the most relevant revenue source for these universities is research, and much part of the research revenue comes from public funding, indicating that any solution to the university funding issue must include active state participation, and that the pursuit of possible funding solutions in international institutions should be done with extreme caution.

Keywords: University Management. University rankings. University financing.

Universidade Federal da Bahia | Escola de Administração | Salvador | BA | Brasil. Contato: tumenas@ufba.br ORCID: https://orcid.org/0000-0001-8327-4215

- Recebido em: 1 de maio de 2020

- Aprovado em: 1 de junho de 2020

DOI: http://dx.doi.org/10.1590/S1414-40772021000100015

Este é um artigo publicado em acesso aberto sob uma licença Creative Commons https://creativecommons.org/licenses/by-nc/4.0/ 


\section{Introdução}

As universidades públicas brasileiras têm sofrido com cortes recorrentes no orçamento, inclusive com riscos de continuidade de suas atividades (KADAMANI; GREVE, 2019). A emenda constitucional 55, conhecida como "PEC do Teto dos gastos", limitou a evolução do orçamento discricionário das universidades públicas apenas à variação da inflação entre um ano e outro. Isso somado à recente maré midiática sobre a suposta "falência" institucional das universidades públicas brasileiras (SERAFIM, 2019). Mesmo assim, as universidades públicas brasileiras têm sido cobradas a mostrar seu impacto na sociedade, aumentar sua internacionalização e melhorar sua posição em rankings internacionais de universidades (GLOBO, 2018) e (DE CASTRO, 2018).

O financiamento da pesquisa pode ter um impacto direto na produção científica, conforme demonstram Kannebley Júnior, Carolo e De Negri (2013), e muitas discussões trazem como solução para os problemas de financiamento das universidades públicas brasileiras fatos isolados das universidades mais conhecidas, notadamente americanas, como fundos patrimoniais, cobrança de mensalidades, doações de ex-alunos e rendas de patentes e serviços. Isso pode ser observado na proposta Future-se (2019) feita pelo Ministério da Educação, onde é dado um peso muito grande aos Fundos Patrimoniais como solução para o financiamento das universidades federais brasileiras. Diversos autores têm estudado os padrões de financiamento das universidades brasileiras, como, por exemplo, Corbucci e Marques (2003), Amaral (2008), Silva (2018), Duarte e Maior de Oliveira (2012), Velloso (2000) e Velloso e Marques (2005). Porém, algumas perguntas permanecem em aberto, como, por exemplo, qual é o padrão de financiamento das universidades mais bem posicionadas nos rankings internacionais? Qual a importância de cada fonte de financiamento dentro de seus respectivos orçamentos? Existe um padrão único entre elas?

Este artigo tem como objetivo elencar quais são as fontes de receitas e qual a importância de cada uma delas no financiamento das universidades melhor posicionadas nos rankings internacionais. $\mathrm{O}$ artigo é divido em cinco partes, sendo a primeira parte essa introdução. Na segunda parte está o levantamento dos Ranking Universitários internacionais. $\mathrm{Na}$ terceira parte está descrita a metodologia utilizada no artigo. Na quarta parte estão os resultados. Na quinta e última parte estão a discussão e a conclusão. 


\section{Ranking universitários internacionais}

Segundo Liu (2009), a história dos rankings universitários internacionais começa efetivamente em junho de 2003 a partir da divulgação do Academic Ranking of World Universities (ARWU). Esse ranking foi criado no contexto do projeto 985, iniciado em 1998 para o estabelecimento de universidades de padrão mundial (world-class universities) na China, com o objetivo de estabelecer um padrão de comparação (benchmark) da Universidade Shanghai Jiao Tong com as universidades estrangeiras.

De acordo com Altbach (2006), os rankings internacionais se tornaram um fator central no cenário das instituições de ensino superior. Para Ordorika e Lloyd (2015), em apenas uma década os rankings universitários internacionais se tornaram mundialmente a métrica de desempenho institucional dominante para os responsáveis por políticas públicas. De acordo com os mesmos autores, isso foi possível graças à uma suposta neutralidade científica desses sistemas de classificação que reforçam o modelo de universidade de pesquisa Anglo-Saxã. Já para Pusser e Marginson (2013), os rankings universitários internacionais são um instrumento de exercício de poder a serviço das normas dominantes no ensino superior global. Taylor e Braddock (2008) levantam a necessidade de distinção entre sistemas de ranking genuínos e ferramentas que servem apenas como um relatório para consumidores. Ordorika e Lloyd (2015) também questionam a possibilidade de comparação entre universidades em ambientes tão díspares como, por exemplo, Shanghai, São Paulo, Cidade do Cabo e Nova Iorque. Porém, apesar dos questionamentos sobre sua validade, os rankings universitários são um meio popular para comparar instituições (BOWMAN; BASTEDO, 2011).

Segundo Valmorbida et al. (2016) e Ordorika e Lloyd (2015), os rankings internacionais mais conhecidos e populares são: Times Higher Education (THE), QS World University (QS) e Academic Ranking of World Universities (ARWU). Segundo Valmorbida et al. (2016), as principais críticas a esses rankings estão relacionadas ao viés para indicadores cuja coleta de dados está disponível, além de serem baseados na opinião de especialistas, favorecendo instituições mais tradicionais; da utilização de um ou poucos indicadores; e ainda por ignorarem a qualidade do ensino e aprendizagem. Rankings internacionais tendem focar no desempenho da pesquisa enquanto os rankings nacionais tendem a focar em indicadores educacionais e institucionais (ÇAKIR et al., 2015).

Com relação à participação das universidades brasileiras, a Universidade de São Paulo (USP) é a melhor classificada nos três rankings. O ranking THE para 2020 possui 46 
universidades brasileiras e a USP está na faixa das Top 251-300, THE (2019). No ranking QS para 2020 existem 20 universidades brasileiras classificadas e a USP está na posição 116, QS (2019). No ranking ARWU para 2019 estão 23 universidades brasileiras e a USP está na faixa 101-150, ARWU (2019). Segundo Santos e Noroña (2016), as universidades brasileiras têm boa pontuação no volume de publicações, porém baixa pontuação nos indicadores que medem impacto por meio de publicações altamente citadas.

Dentre as principais características de cada um dos três rankings, podemos citar:

\section{a) Times Higher Education}

Segundo THE (2019), o ranking Times Higher Education (THE) avalia as universidades com base em cinco áreas distintas, com seus indicadores Ensino (o ambiente de ensino); Pesquisa (volume, receita e reputação); Citações (influência da pesquisa); Perfil Internacional (equipe, estudantes e pesquisa); e Receita da Indústria (transferência de conhecimento). Os pesos de cada área na avaliação final são dados pelos os respectivos pesos, respectivamente: $30 \%, 30 \%, 30 \%, 7,5 \%$ e $2,5 \%$.

\section{b) QS World University}

De acordo com QS (2019), o QS World University Rankings avalia as universidades usando seis indicadores de desempenho, cada um com uma ponderação diferente no cálculo da pontuação geral. Esses indicadores são: reputação acadêmica (40\%), reputação para o empregador (10\%), razão aluno/professor (20\%), citações de pesquisa por professor (20\%), proporção de professores internacionais (5\%).

\section{c) Academic Ranking of World Universities (ARWU)}

Segundo ARWU (2019), o ARWU, também conhecido como Shanghai Ranking, usa seis indicadores objetivos para classificar as universidades mundiais, incluindo o número de ex-alunos vencedores do Prêmio Nobel e Medalha Fields e membros do corpo docente que obtiverem tais prêmios, o número de pesquisadores altamente citados selecionados pela Thomson Reuters, o número de artigos publicados nas revistas de grande prestígio, como Nature e Science, o número de artigos indexados no Science Citation Index - Expanded e Social Sciences Citation Index, e desempenho docente per capita da universidade. 
Para este trabalho serão consideradas as 10 melhores universidades dos rankings THE, QS, ARWU para o ano de 2020. Na tabela 1 temos a lista dessas universidades nos três rankings. Apesar de serem rankings diferentes, há uma convergência nas primeiras posições dos três rankings. As 10 primeiras posições nos três rankings são ocupadas por um grupo de 14 universidades distintas. Dessas universidades, 4 são inglesas (Cambridge, Imperial College, Oxford e UCL), 1 suíça (ETH) e 9 norte-americanas (Berkeley, Caltech, Chicago, Columbia, Harvard, MIT, Princeton, Stanford, Yale)

Tabela 1 - Melhores Universidades do Mundo

\begin{tabular}{cccc}
\hline Posição & ARWU & THE & QS \\
\hline 1 & Harvard & Oxford & MIT \\
2 & Stanford & Caltech & Stanford \\
3 & Cambridge & Cambridge & Harvard \\
4 & MIT & Stanford & Oxford \\
5 & Berkeley & MIT & Caltech \\
6 & Princeton & Princeton & ETH \\
7 & Oxford & Harvard & Cambridge \\
8 & Columbia & Yale & UCL \\
9 & Caltech & Chicago & Imperial College \\
10 & Chicago & Imperial College & Chicago \\
\hline
\end{tabular}

Fonte: Elaboração própria a partir de ARWU (2019), THE (2019) e QS (2019).

\section{Metodologia}

O problema de pesquisa deste artigo é elencar as fontes de receitas das universidades melhor posicionadas nos rankings universitários internacionais e determinar a importância de cada uma delas em seus orçamentos. Para tanto, foram avaliados os relatórios anuais dessas universidades, disponibilizados em seus respectivos sítios eletrônicos. Os sítios eletrônicos serão consultados no ano de 2019. Nos relatórios anuais estão informados os demonstrativos financeiros, com informações de receitas, despesas, ativos e passivos. Para cada universidade foram analisados os relatórios anuais e seus respectivos demonstrativos financeiros dos últimos cinco anos, de 2014 até 2018, o que totaliza 70 relatórios anuais para todas as 14 universidades consideradas. No caso de Berkeley, além de ser a única universidade pública da lista, a universidade faz parte do University of California System, com 10 campi em diferentes localidades no estado da Califórnia. Os relatórios anuais são disponibilizados de maneira consolidada para o University of California System, com os 10 campi, e não apenas de Berkeley.

Após uma avaliação preliminar dos 70 relatórios anuais, as receitas foram classificadas em cinco grandes categorias: 
- Mensalidades. Nessa categoria estão as linhas dos demonstrativos financeiros que se referem a "Tuition Fees" e "Education Contracts" e que já estão descontados das bolsas estudantis ("net of student financial aid"). Algumas universidades divulgam separadamente tanto a informação das mensalidades como das bolsas estudantis, porém outras divulgam apenas os valores já descontados das bolsas. Para manter o mesmo padrão de comparação, serão considerados apenas os valores já descontados das bolsas estudantis;

- Doações e Fundos Patrimoniais. Nessa categoria estão agrupadas as linhas de receitas nos demonstrativos financeiros categorizadas como "Gifts and Pledges", "Investment Income", "Endowment", "Donations and Bequests" e "Donations";

- Financiamento para Pesquisa: Nessa categoria estão categorias como "Funding Body Grants", "Research Grants and Contracts", "Grants and contract income”, além de nomes específicos de financiamentos para cada universidade, como "Federal Pell grants" e "Swiss National Science Foundation". Nem todas as universidades informam, nas notas explicativas dos demonstrativos financeiros, se o financiamento é público ou privado, portanto iremos considerar apenas o valor total do financiamento para pesquisa, sem distinção entre financiamento público ou privado;

- Serviços: Algumas universidades oferecem serviços que são uma fonte de receita relevante, como serviços médicos (Stanford, Columbia, Berkeley, Yale), serviços de editora (Oxford) e de testes (Cambridge). Essas receitas estão agrupadas nessa categoria;

- Outros: Nessa categoria estão as linhas contábeis definidas como "Other Income", que comumente tem pouca relevância no total de receitas das universidades, e receitas relevantes específicas de algumas universidades que não são classificadas nas categorias previamente estabelecidas.

Foram calculadas, para cada universidade dentro do período de 2014 a 2018, as proporções de cada categoria na receita total das universidades. Essas informações foram consolidadas nas medidas estatísticas descritivas média, desvio padrão e coeficiente de variação $(\mathrm{CV})$. A média serve para avaliar a importância da categoria na receita total. $\mathrm{O}$ desvio padrão serve para avaliar o quanto a proporção oscila no decorrer do período considerado. O CV serve para avaliar se a oscilação é grande ou não, sendo calculada como a razão percentual entre o desvio padrão e a média. 


\section{Resultados}

\subsection{Mensalidades}

Na tabela 2 temos a média, o desvio padrão e o coeficiente de variação $(\mathrm{CV})$ da proporção das mensalidades nas receitas nos últimos 5 anos para cada uma das universidades.

Tabela 2 - Proporção das Mensalidades nas Receitas no período de 2014 a 2018

\begin{tabular}{lccc}
\hline & Média & Desvio Padrão & CV \\
\cline { 2 - 3 } Berkeley & $13,28 \%$ & $0,28 \%$ & $1,77 \%$ \\
Caltech & $1,60 \%$ & $0,19 \%$ & $11,44 \%$ \\
Cambridge & $14,67 \%$ & $1,01 \%$ & $6,90 \%$ \\
Chicago & $10,68 \%$ & $0,85 \%$ & $7,95 \%$ \\
Columbia & $23,08 \%$ & $0,31 \%$ & $1,34 \%$ \\
ETH & $1,27 \%$ & $0,03 \%$ & $2,18 \%$ \\
Harvard & $20,92 \%$ & $0,40 \%$ & $1,92 \%$ \\
Imperial College & $25,19 \%$ & $2,61 \%$ & $10,36 \%$ \\
MIT & $10,06 \%$ & $0,24 \%$ & $2,40 \%$ \\
Oxford & $21,07 \%$ & $2,41 \%$ & $11,45 \%$ \\
Princeton & $6,33 \%$ & $0,63 \%$ & $9,99 \%$ \\
Stanford & $6,09 \%$ & $0,42 \%$ & $6,94 \%$ \\
UCL & $31,84 \%$ & $3,33 \%$ & $10,46 \%$ \\
Yale & $9,52 \%$ & $0,15 \%$ & $1,61 \%$ \\
\hline \multicolumn{1}{c}{ Média } & $\mathbf{1 3 , 8 6 \%}$ & $\mathbf{0 , 9 5 \%}$ & $\mathbf{6 , 4 2 \%}$ \\
\hline
\end{tabular}

Fonte: Elaboração própria a partir dos dados dos relatórios financeiros das universidades.

É possível notar que não existe um padrão único na participação das mensalidades nas receitas totais das universidades. Temos tanto universidades como Caltech e ETH, nas quais essa participação é baixa, com $1,60 \%$ e $1,27 \%$ respectivamente, como também universidades como Columbia e UCL, nas quais a participação é alta, com 23,08\% e 31,84\% respectivamente. A participação das mensalidades nas receitas é estável no período considerado, com os desvios padrão de todas as universidades sendo bem menores que as médias durante os anos analisados. Isso pode ser avaliado na coluna do CV. A média da participação das mensalidades no orçamento de todas as universidades, no período de 2014 a 2018, é de 13,86\% e o CV médio para todas as universidades é de apenas $6,42 \%$.

\subsection{Doações e fundos patrimoniais}

$\mathrm{Na}$ tabela 3 estão os dados relacionados à participação das Doações e Fundos Patrimoniais nas receitas das universidades. 
Tabela 3 - Proporção das Doações e Fundos Patrimoniais nas Receitas no período de 2014 a 2018

\begin{tabular}{lccc}
\hline & Média & Desvio Padrão & CV \\
\cline { 2 - 3 } Berkeley & $4,53 \%$ & $0,18 \%$ & $4,08 \%$ \\
Caltech & $7,29 \%$ & $1,96 \%$ & $26,85 \%$ \\
Cambridge & $4,36 \%$ & $1,57 \%$ & $36,00 \%$ \\
Chicago & $14,41 \%$ & $0,51 \%$ & $3,52 \%$ \\
Columbia & $12,91 \%$ & $0,11 \%$ & $0,84 \%$ \\
ETH & $4,52 \%$ & $1,78 \%$ & $39,37 \%$ \\
Harvard & $47,69 \%$ & $0,66 \%$ & $1,38 \%$ \\
Imperial College & $4,76 \%$ & $2,50 \%$ & $52,55 \%$ \\
MIT & $27,51 \%$ & $2,49 \%$ & $9,06 \%$ \\
Oxford & $6,72 \%$ & $2,35 \%$ & $34,90 \%$ \\
Princeton & $57,81 \%$ & $4,91 \%$ & $8,49 \%$ \\
Stanford & $18,34 \%$ & $0,80 \%$ & $4,35 \%$ \\
UCL & $1,86 \%$ & $0,77 \%$ & $41,48 \%$ \\
Yale & $37,65 \%$ & $0,41 \%$ & $1,08 \%$ \\
\hline \multicolumn{1}{c}{ Média } & $\mathbf{1 7 , 8 5 \%}$ & $\mathbf{1 , 4 9 \%}$ & $\mathbf{1 8 , 8 9 \%}$ \\
\hline
\end{tabular}

Fonte: Elaboração própria a partir dos dados dos relatórios financeiros das universidades.

Apesar da média geral ser de $17,85 \%$, temos que nas universidades europeias da lista (Cambridge, ETH, Oxford, UCL) a participação média de doações e fundos patrimoniais nas receitas é menor que 5\% para todas, e o CV é bem alto. A oscilação dessa categoria nas receitas não é pequena, sendo o CV da participação de Doações e Fundos Patrimoniais nas receitas quase três vezes maior que o CV da participação das Mensalidades nas receitas. Nas universidades norte americanas, a participação é maior nas universidades localizadas no Nordeste, notadamente Harvard, Yale e Princeton, onde mais de um terço das receitas dos orçamentos são provenientes de doações e resultados financeiros dos fundos patrimoniais. Já para universidades localizadas na costa leste, como Berkeley e Caltech, a média é menor que $10 \%$, com exceção de Stanford onde a participação média das mensalidades nas receitas é de $18,34 \%$.

\subsection{Pesquisa}

$\mathrm{Na}$ tabela 4 estão os dados relacionados à proporção das Pesquisas nas receitas das universidades. 
Tabela 4 - Proporção das Pesquisas nas Receitas no período de 2014 a 2018

\begin{tabular}{lccc}
\hline & Média & Desvio Padrão & CV \\
\cline { 2 - 3 } Berkeley & $22,25 \%$ & $1,82 \%$ & $8,20 \%$ \\
Caltech & $88,94 \%$ & $2,24 \%$ & $2,52 \%$ \\
Cambridge & $37,96 \%$ & $1,27 \%$ & $3,35 \%$ \\
Chicago & $13,68 \%$ & $3,03 \%$ & $22,16 \%$ \\
Columbia & $35,12 \%$ & $0,93 \%$ & $2,64 \%$ \\
ETH & $18,28 \%$ & $0,52 \%$ & $2,83 \%$ \\
Harvard & $17,90 \%$ & $0,48 \%$ & $2,70 \%$ \\
Imperial College & $54,55 \%$ & $4,89 \%$ & $8,99 \%$ \\
MIT & $48,23 \%$ & $0,87 \%$ & $1,81 \%$ \\
Oxford & $56,37 \%$ & $1,68 \%$ & $2,98 \%$ \\
Princeton & $22,28 \%$ & $0,47 \%$ & $2,13 \%$ \\
Stanford & $15,27 \%$ & $0,55 \%$ & $3,58 \%$ \\
UCL & $50,98 \%$ & $2,69 \%$ & $5,27 \%$ \\
Yale & $20,78 \%$ & $0,46 \%$ & $2,22 \%$ \\
\hline \multicolumn{1}{c}{ Média } & $\mathbf{3 5 , 6 3 \%}$ & $\mathbf{2 , 0 5 \%}$ & $\mathbf{6 , 0 3 \%}$ \\
\hline
\end{tabular}

Fonte: Elaboração própria a partir dos dados dos relatórios financeiros das universidades.

A média geral para a participação das receitas de pesquisas é de 35,63\% e o CV médio para todas as universidades, no período de 2014 a 2018, é de apenas 6,03\%. A participação das receitas de pesquisas também não apresenta um padrão único entre as universidades consideradas. Em um extremo temos a universidade de Chicago que tem uma participação de receitas de pesquisas média de $13,68 \%$, enquanto no outro extremo temos Caltech onde essa participação é de $88,94 \%$. Um ponto importante é que nem todas as universidades detalham a origem da receita de pesquisa em seus relatórios, se são fontes públicas ou privadas. Nas universidades que detalham alguma origem das receitas de pesquisa temos os seguintes casos:

- Berkeley: 69\% das receitas de pesquisa vem das linhas "Federal Pell Grants", "Federal grants and contracts net revenue" e "Department of Energy laboratories";

- Caltech: a alta participação de receitas de pesquisa se dá pela principal linha de receitas da universidade ser o Jet Propulsion Laboratory, um laboratório de pesquisa financiado pelo governo americano em conjunto com a NASA. Em 2018, por exemplo, as receitas desse laboratório representaram mais de $84 \%$ das receitas totais da universidade;

- Chicago: $55 \%$ das receitas de pesquisa vem de "Government grants and contracts";

- Columbia: $54 \%$ das receitas de pesquisa vem de "Government grants and contracts";

- ETH: apenas 17\% das receitas de pesquisa vem de "Industry-oriented research (private sector)", enquanto os $83 \%$ restantes provenientes de diversas fontes governamentais, como o governo suíço, União Europeia, cantões da Suiça e o município de Zurique; 
- Harvard: $60 \%$ das receitas de pesquisa vem da linha "Federal Government" enquanto o restante está na descrição como "Non federal sponsors", o que não exclui outros entes governamentais, como, por exemplo, governo estadual ou agências governamentais;

- MIT: 56\% das receitas de pesquisa vem apenas do Lincoln Laboratory, um centro de pesquisa e desenvolvimento do departamento de Defesa norte americano. Apenas $7,77 \%$ das receitas de pesquisa vem da indústria ("Non-federally sponsored Industry");

- Princeton: 74\% das receitas de pesquisa estão na linha "Government Grants and Contracts";

- Stanford: $32 \%$ das receitas de pesquisa vem apenas do SLAC - National Accelerator Laboratory do Departamento de Energia norte americano.

Ou seja, o financiamento público tem um papel central na pesquisa nas universidades bem posicionadas nos rankings internacionais, seja através de bolsas de pesquisa ou através de laboratórios mantidos diretamente pelos governos.

\subsection{Serviços}

Na tabela 5 estão os dados relacionados à participação das receitas de Serviços nas receitas das universidades.

Tabela 5 - Proporção de Serviços nas Receitas no período de 2014 a 2018

\begin{tabular}{lccc}
\hline & Média & Desvio Padrão & CV \\
\cline { 2 - 4 } Berkeley & $47,14 \%$ & $1,64 \%$ & $3,49 \%$ \\
Caltech & $1,12 \%$ & $0,15 \%$ & $13,03 \%$ \\
Cambridge & $35,63 \%$ & $2,50 \%$ & $7,00 \%$ \\
Chicago & $54,26 \%$ & $2,32 \%$ & $4,27 \%$ \\
Columbia & $28,87 \%$ & $0,69 \%$ & $2,39 \%$ \\
ETH & - & - & - \\
Harvard & - & - & - \\
Imperial College & - & - & - \\
MIT & $8,45 \%$ & $1,06 \%$ & $12,50 \%$ \\
Oxford & $32,19 \% *$ & - & - \\
Princeton & $5,41 \%$ & $0,65 \%$ & $11,94 \%$ \\
Stanford & $53,14 \%$ & $2,23 \%$ & $4,20 \%$ \\
UCL & - & - & - \\
Yale & $23,87 \%$ & $0,92 \%$ & $3,83 \%$ \\
\hline \multicolumn{1}{c}{ Média } & $\mathbf{2 8 , 6 5 \%}$ & $\mathbf{1 , 3 5 \%}$ & $\mathbf{6 , 9 6 \%}$ \\
\hline
\end{tabular}

Fonte: Elaboração própria a partir dos dados dos relatórios financeiros das universidades.

* informação específica de 2018 
Algumas universidades não possuem receitas classificadas como serviços, como ETH, Harvard, Imperial College, e UCL. Uma explicação para esse fenômeno pode ser que as receitas de serviços não são relevantes para divulgarem informações específicas e, consequentemente, essas receitas são classificadas na categoria "Outros". Para as universidades que possuem receitas como serviços, assim como nas outras categorias de receitas, não existe um padrão único para a participação das receitas de serviços. Nas universidades que divulgaram os dados de receitas de serviços, a participação média geral de serviços é de 28,65\% e o CV é de 6,96\%. Nas universidades de Berkeley, Chicago, Columbia, Stanford, Yale a receita de serviços tem origem principalmente em serviços médicos pelas universidades. Cambridge tem como principal receita de serviços os testes de proficiência de língua inglesa. Oxford possui serviços de publicação (editora) como fonte de receitas de serviços, que está disponível apenas nos demonstrativos de 2018. A participação das receitas de serviços de 32,19\% se refere a esse período específico. Nos períodos anteriores, as receitas da editora não eram consideradas nos demonstrativos financeiros de Oxford.

\subsection{Outros}

Na tabela 6 estão os dados relacionados à participação das receitas classificadas como Outros nas receitas das universidades.

Tabela 6 - Proporção de Outros nas Receitas no período de 2014 a 2018

\begin{tabular}{lccc}
\hline & Média & Desvio Padrão & CV \\
\cline { 2 - 3 } Berkeley & $12,80 \%$ & $0,17 \%$ & $1,29 \%$ \\
Caltech & $1,12 \%$ & $0,55 \%$ & $49,24 \%$ \\
Cambridge & $6,69 \%$ & $0,93 \%$ & $13,86 \%$ \\
Chicago & $9,04 \%$ & $0,47 \%$ & $5,23 \%$ \\
Columbia & $0,01 \%$ & $0,02 \%$ & $115,44 \%$ \\
ETH & $64,20 \%$ & $1,24 \%$ & $1,94 \%$ \\
Harvard & $13,49 \%$ & $0,50 \%$ & $3,73 \%$ \\
Imperial College & $15,60 \%$ & $1,77 \%$ & $11,32 \%$ \\
MIT & $2,86 \%$ & $0,71 \%$ & $24,84 \%$ \\
Oxford & $15,06 \%$ & $4,95 \%$ & $32,88 \%$ \\
Princeton & $8,17 \%$ & $3,37 \%$ & $41,28 \%$ \\
Stanford & $7,16 \%$ & $0,62 \%$ & $8,64 \%$ \\
UCL & $15,32 \%$ & $2,68 \%$ & $17,50 \%$ \\
Yale & $8,18 \%$ & $0,34 \%$ & $4,12 \%$ \\
\hline \multicolumn{1}{c}{ Média } & $\mathbf{1 2 , 8 4 \%}$ & $\mathbf{1 , 3 1 \%}$ & $\mathbf{2 3 , 6 6 \%}$ \\
\hline
\end{tabular}

Fonte: Elaboração própria a partir dos dados dos relatórios financeiros das universidades.

No geral, a participação de "Outros" nas receitas das universidades é baixa e sua variação é alta, com um CV médio de 23,66\%. A exceção na tabela, ETH, com participação 
média de $64,20 \%$ é devida ao subsídio direto do governo à Universidade (e que não estava classificada em nenhuma outra categoria anterior de receitas). Se não considerarmos a ETH, a média geral da participação de "Outros" nas receitas das universidades cai de 12,84\% para $8,85 \%$.

\section{Discussão e conclusão}

Comparando as médias gerais de participação nas receitas das cinco categorias, a maior fonte de receitas são os financiamentos de Pesquisa, com 36\%, seguidos por Serviços (normalmente serviços médicos) com 28\%, depois por Doações e FP, com 18\%. Mensalidades e Outros respondem por aproximadamente $14 \%$. Isso pode ser observado no Gráfico 1.

Gráfico 1 - Participação média nas receitas das universidades

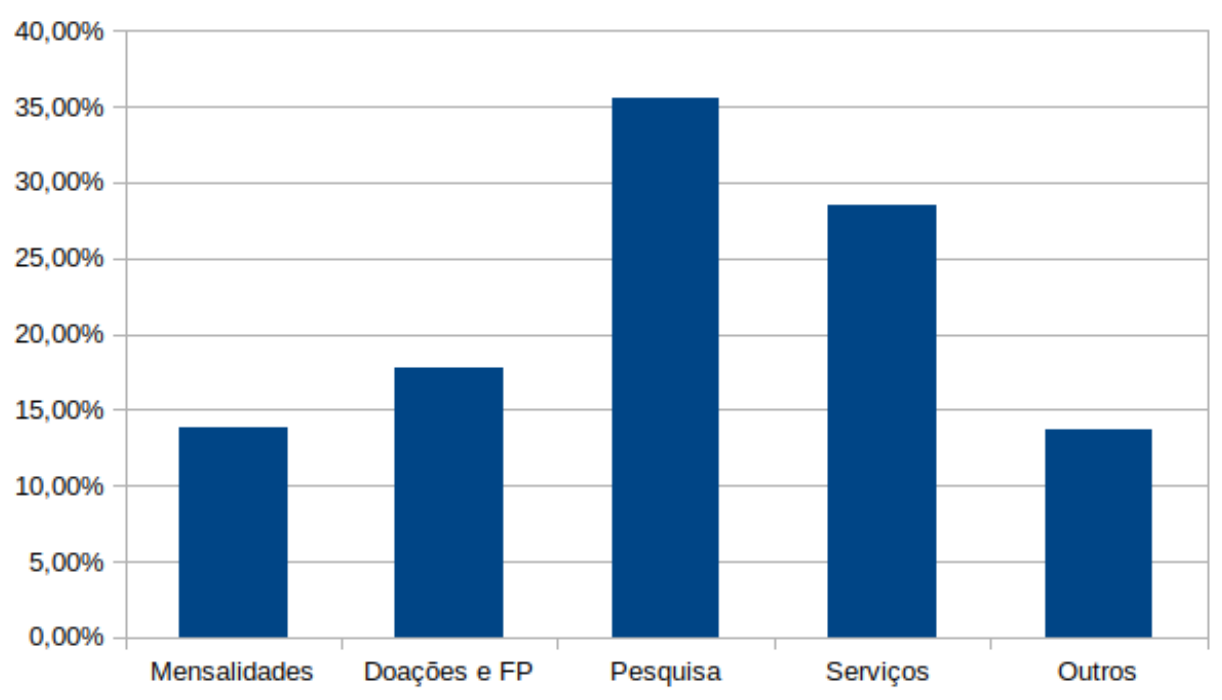

Fonte: Elaboração própria.

Conforme observado no Gráfico 1 na seção anterior, a maior fonte de receita das universidades mais bem colocadas nos rankings internacionais são os financiamentos de Pesquisa, seguidos por receitas de Serviços prestados pelas universidades. Porém, essas proporções têm de ser avaliadas com cuidado, pois não existe um padrão único entre as universidades avaliadas. Um exemplo é a participação de Doações e Fundos Patrimoniais na receita das universidades que, apesar da proporção média estar em terceiro lugar, sendo responsável por $18 \%$ das receitas, possui uma alta disparidade entre as universidades. Universidades localizadas na Europa (Cambridge, ETH, Imperial College, Oxford, UCL) têm proporção de Doações e Fundos Patrimoniais muito baixa em comparação à proporção de 
Doações e Fundos Patrimoniais das universidades norte americanas da lista. Mesmo na tradicional Universidade de Cambridge, fica evidente no relatório de 2018 que essa categoria de receita ainda é muito incipiente e resultado de poucos doadores ("A significant strategic focus on closing eight-figure gifts also proved successful. Three gifts of more than $110 \mathrm{~m}$ and one at $£ 9.25 m$ totalling $£ 124 m$ accounted for over $70 \%$ of total funds raised this year”). Também entre as universidades norte-americanas não existe um padrão comum. Existem universidades com altos fundos patrimoniais e outras com participação modesta de doações e fundos patrimoniais nas receitas. Em Berkeley o foco das doações é, segundo relatório anual de 2018, um meio de prover flexibilidade nas iniciativas acadêmicas ("Gifts to the University allow crucial flexibility to faculty for support of their fundamental activities or new academic initiatives") e não uma fonte central de receita para as operações cotidianas da universidade. Fundos patrimoniais, portanto, não são uma forma unanimemente relevante de receita mesmo nas melhores universidades do mundo, por isso sua utilização no Brasil deve ser olhada com cautela. Como experiência brasileira em fundos patrimoniais, existe o Fundo Patrimonial Amigos da Poli (FPAP) da Escola Politécnica de Universidade de São Paulo. Fundado em 2012 e associado à uma escola que possui como ex-alunos grandes nomes do setor industrial e financeiro, o fundo tem um patrimônio de cerca de $\mathrm{R} \$ 23$ milhões (dos quais serão utilizados apenas os rendimentos financeiros) e apoia projetos de iniciativa acadêmica, FPAP (2019). Isso indica que fundos patrimoniais no Brasil têm uma proximidade maior dos objetivos do fundo patrimonial de Berkeley do que se tornar uma fonte de recursos para as operações da universidade, como Harvard e Yale.

Outro ponto de atenção é a importância do financiamento público, independentemente se a instituição é pública ou privada. Nas universidades que possuem algum detalhamento das receitas de pesquisas podemos ver a elevada participação do financiamento público. Seja em bolsas, seja em laboratórios mantidos diretamente pelo governo nas universidades, como Lincoln Lab no MIT, JPL na Caltech e SLAC em Stanford, ou seja, em financiamento direto como na ETH. As mensalidades também não têm um padrão único nas receitas das universidades. Com algumas exceções, as mensalidades têm baixa a participação na receita das universidades líderes nos rankings internacionais. Ademais, algumas universidades têm nas receitas de serviços uma importante fonte de recursos, principalmente com serviços médicos.

Em suma, não existe um padrão nas receitas das universidades melhor posicionadas nos rankings universitários internacionais, com as universidades tendo perfis diferentes de atuação 
e receitas, o que inviabiliza a busca por uma "solução ótima" de financiamento para as universidades públicas brasileiras. Os resultados aqui expostos vão em linha com Millot (2015), que afirma seria mais útil para decisões de políticas públicas a comparação de sistemas universitários, e não apenas a comparação de instituições isoladamente. Portanto, antes da questão financeira, é imperativo estabelecer o papel das universidades públicas brasileiras no cenário nacional e determinar quais os sistemas universitários que devemos ter como referência, e não apenas buscar inspiração em instituições isoladas em rankings internacionais.

\section{Referências}

ALTBACH, Philip. The dilemmas of ranking. International higher education, United States, n. 42, 2006.

AMARAL, Nelson Cardoso. Autonomia e financiamento das IFES: desafios e ações. Avaliação, Campinas; Sorocaba, v. 13, n. 3, p. 647-680, 2008.

ARWU - Academic Ranking of World Universities. China, 2019. Disponível em: http://www.shanghairanking.com/arwu2019.html. Acesso em: 14 abr. 2020.

BERKELEY. Annual Financial Reports. United States, 2019. Disponível em: https://www.ucop.edu/financial-accounting/financial-reports/annual-financial-reports.html. Acesso em: 14 abr. 2020.

BOWMAN, Nicholas A.; BASTEDO, Michael N. Anchoring effects in world university rankings: exploring biases in reputation scores. Higher Education, Amstedam, v. 61, n. 4, p. 431-444, 2011.

CALTECH. Financial statements. United States, 2019. Disponível em: http://www.businessandfinance.caltech.edu/finstatements. Acesso em: 14 abr. 2020.

CAMBRIDGE. Annual report. United Kingdom, 2019. Disponível em: https://www.cam.ac.uk/annual-report. Acesso em: 14 abr. 2020.

ÇAKIR, Murat Perit et al. A comparative analysis of global and national university ranking systems. Scientometrics, Hungary, v. 103, n. 3, p. 813-848, 2015.

CHICAGO. Financial statements. United States, 2019. Disponível em: https://finserv.uchicago.edu/reporting/statements.shtml. Acesso em: 14 abr. 2020.

COLUMBIA. Financial reports. United States, 2019. Disponível em: https://finance.columbia.edu/content/columbia-university-financial-reports. Acesso em: 14 abr. 2020. 
CORBUCCI, Paulo Roberto; MARQUES, Paulo Marcello Fonseca. Fontes de financiamento das instituições federais de ensino superior: um estudo sobre a Universidade de Brasília. Brasília: UnB, 2003.

DUARTE, Cássia Soares; MAIOR DE OLIVEIRA, Tatiana Souto. O financiamento das instituições federais de ensino superior: o caso da Universidade Federal de Goiás. Revista Organização Sistêmica, Curitiba, v. 2, n. 1, p. 102-118, 2012.

DE CASTRO, Fábio. Universidades Brasileiras caem novamente em ranking internacional. São Paulo, 2018. Disponível em:

https://educacao.estadao.com.br/noticias/geral,universidades-brasileiras-caem-novamente-emranking-internacional,70002301522. Acesso em: 14 abr. 2020.

ETH. Annual report. Switzerland, 2019. Disponível em:

https://ethz.ch/en/the-eth-zurich/information-material/annual-report.html. Acesso em: 14 abr. 2020.

FPAP - Fundo Patrimonial Amigos da Poli. São Paulo, 2019. Disponível em: https://www.amigosdapoli.com.br/. Acesso em: 14 abr. 2020.

FUTURE-SE. Programa Future-se. Brasília, 2019. Disponível em: https://isurvey.cgee.org.br/future-se/. Acesso em: 14 abr. 2020.

GLOBO. Número de universidades brasileiras entre as melhores do mundo cai pelo segundo ano consecutivo. Rio de Janeiro, 2018. Disponível em:

https://oglobo.globo.com/sociedade/educacao/numero-de-universidades-brasileiras-entre-asmelhores-do-mundo-cai-pelo-segundo-ano-consecutivo-23104006. Acesso em: 14 abr. 2020.

HARVARD. Annual report. United States, 2019. Disponível em: https://finance.harvard.edu/annual-report. Acesso em: 14 abr. 2020.

IMPERIAL COLLEGE. Annual report. United Kingdom, 2019. Disponível em: https://www.imperial.ac.uk/finance/annual-report. Acesso em: 14 abr. 2020.

KADAMANI, Rosine, GREVE, Fabíola. Inovar? Que tal sobreviver primeiro? Época Negócios, São Paulo, 2019. Disponível em:

https://epocanegocios.globo.com/colunas/noticia/2019/09/inovar-que-tal-sobreviverprimeiro.html. Acesso em: 14 abr. 2020.

KANNEBLEY JÚNIOR, Sérgio; CAROLO, Murilo Damião; DE NEGRI, Fernanda. Impacto dos Fundos Setoriais sobre a produtividade acadêmica de cientistas universitários. Estudos Econômicos, São Paulo, v. 43, n. 4, p. 647-685, 2013.

LIU, Nian Cai. The story of academic ranking of world universities. International Higher Education, United States, n. 54, 2009. 
MILLOT, Benoit. International rankings: Universities vs. higher education systems.

International Journal of Educational Development, United Kingdom, v. 40, p. 156-165, 2015.

MIT. Report of the Treasurer. United States, 2019. Disponível em:

https://vpf.mit.edu/about-vpf/publications. Acesso em: 14 abr. 2020.

ORDORIKA, Imanol; LLOYD, Marion. International rankings and the contest for university hegemony. Journal of Education Policy, United Kingdom, v. 30, n. 3, p. 385-405, 2015.

OXFORD. Financial statements. United Kingdom, 2019. Disponível em:

https://www.ox.ac.uk/about/organisation/finance-and-funding/archive-of-financial-statements. Acesso em: 14 abr. 2020.

PRINCETON. Report of the Treasurer. United States, 2019. Disponível em: https://finance.princeton.edu/report-treasurer. Acesso em: 14 abr. 2020.

PUSSER, Brian; MARGINSON, Simon. University rankings in critical perspective. The Journal of Higher Education, United States, v. 84, n. 4, p. 544-568, 2013.

QS. QS World University Rankings London, 2019. Disponível em:

https://www.topuniversities.com/university-rankings/world-university-rankings/2020. Acesso em: 14 abr. 2020.

SANTOS, Solange Maria; NOROÑA, Daisy Pires. O desempenho das universidades brasileiras em rankings internacionais. Em Questão, Porto Alegre, v. 22, n. 2, p. 186-219, 2016.

SERAFIM, Milena Pavan. Compreendendo o atual momento da educação superior brasileira: cultivando a mais lúcida consciência. Avaliação, Campinas; Sorocaba, v. 24, n. 2, p. 351-356, 2019. Disponível em: https://www.scielo.br/scielo.php?script=sci_arttext\&pid=S141440772019000200351. Acesso em: 14 abr. 2020.

SILVA, Marta Rosa Farias de Almeida Miranda. Investimentos públicos em educação superior na Bahia: o orçamento das Universidades Estaduais Baianas/Public investments in higher education in Bahia: the budget of Baian State Universities. Brazilian Journal of Development, Curitiba, v. 5, n. 2, p. 979-995, 2018.

STANFORD. Annual report. United States, 2019. Disponível em: https://annualreport.stanford.edu/. Acesso em: 14 abr. 2020.

TAYLOR, Paul; BRADDOCK, Richard. International university ranking systems and the idea of university excellence. Journal of Higher Education Policy and Management, United Kingdom, v. 29, n. 3, p. 245-260, 2008.

THE. World University Rankings. United Kingdom, 2019. Disponível em: https://www.timeshighereducation.com/world-university-rankings/2020/worldranking\#!/page/0/length/25/sort_by/rank/sort_order/asc/cols/stats. Acesso em: 14 abr. 2020. 
UCL. Annual report and financial statements. United Kingdom, 2019. Disponível em: https://www.ucl.ac.uk/finance/home/annual-report-and-financial-statements. Acesso em: 14 abr. 2020.

VALMORBIDA, Sandra Mara Iesbik et al. Rankings universitários mundiais. Que dizem os estudos internacionais? REICE. Revista Iberoamericana sobre Calidad, Eficacia y

Cambio en Educación, Madrid, v. 14, n. 2, p. 5-29, 2016.

VELLOSO, Jacques. Universidade na América Latina: rumos do financiamento. Cadernos de Pesquisa, São Paulo, n. 110, p. 39-66, 2000.

VELLOSO, Jacques; MARQUES, Paulo Marcello F. Recursos próprios da UNB, o financiamento das IFES e a reforma da educação superior. Educação \& Sociedade, Campinas, v. 26, n. 91, p. 655-680, 2005.

YALE. Financial reports. United States, 2019. Disponível em:

https://your.yale.edu/work-yale/financial-management/accounting/financial-reports. Acesso em: 14 abr. 2020. 